\title{
Are Antarctic Nanohaloarchaeota emerging viral lineages?
}

\author{
Claudiu I. Bandea \\ Address: Centers for Disease Control and Prevention, 1600 Clifton Rd. Atlanta, GA 30333, USA \\ Email: cbandea@cdc.gov
}

\begin{abstract}
$\underline{\text { Abstract }}$
A recent report in PNAS that Candidatus Nanohaloarchaeum antarcticus requires haloarchaeon Halorubrum lacusprofundi for growth expands the list of known symbiotic or parasitic associations between the members of DPANN archaea, which are relatively small cells with reduced genomes and limited metabolic capacity, and freeliving archaea. In line with previous studies addressing the enigmatic mechanism(s) for the transfer of metabolites from Ignicoccus hospitalis to Nanoarchaeum equitans, this new study presents additional evidence supporting a direct cytoplasmic connection facilitated by the fusion of parasite's membrane with that of its host. Here I show that this novel mechanism for accessing the host resources by a membrane fusing mechanism, which eliminates the need for sophisticated multivalent transport systems, is fundamentally similar to that employed by several viral lineages. These new findings support an evolutionary model on the origin of incipient viral lineages from parasitic cellular lineages that started their parasitic life cycle by fusing with their host cells.
\end{abstract}

In PNAS, Hamm et al. (1) report that Candidatus Nanohaloarchaeum antarcticus (Ca. Nha. antarcticus) requires haloarchaeon Halorubrum lacusprofundi (Hrr. lacusprofundi) for growth. Their study expands the list of known symbiotic/parasitic associations between the members of DPANN archaea, which are relatively small cells with reduced genomes and limited metabolic capacity, and free-living archaea (2).

With the discovery of Nanoarchaeum equitans as an ectoparasite of the archaeon host Ignicoccus hospitalis, one of the most pressing and fascinating questions concerned the mechanism(s) for the transfer of metabolites and other components (3). After dozens of studies, there is convincing evidence that the mechanism involves direct cytoplasmic connection between $N$. equitans and $I$. hospitalis, facilitated by the fusion of their membranes (3, Fig. 1). Remarkably, Hamm et al. present evidence not only for the dependence of $\mathrm{Ca}$. Nha. antarcticus on its haloarchaeon host, but also for the membrane/cellular fusion process as a putative transfer mechanism (Fig. 1).

The fusion of a parasitic cellular organism with its host cell increases the parasite's access to the host resources, without the need for sophisticated transport systems (3; Fig. 1). More significantly, though, the parasite can access to the host's information processing machineries, including the ribosomes, which creates unique parasitic and evolutionary opportunities not easily accessible for parasites that maintain an integral membrane and cellular structure throughout their life cycle.
This scenario is in line with a radical hypothesis on the evolutionary origin of incipient viral lineages from parasitic cellular lineages that start their parasitic life cycle by fusing with their host cells (4-6; Fig. 1). A critical premise for this evolutionary model is that only parasitic lineages that have a cellular and molecular composition compatible with that of their host cells (e.g. an archaeal lineage parasitizing another archaeal lineage) have the opportunity to evolve into a viral lineage (4-6); this implies that a bacterial lineage parasitizing an eukaryal host cell, for example, might not be able to evolve into a viral lineage regardless of the degree of their genome/proteome reduction. Another intriguing inference from this model is that numerous parasitic cellular lineages evolved into viral lineages throughout the history of life and that this process might still be active. So, are Nanohaloarchaeota emerging viral lineages?

The question "What is a virus?" had puzzled scientists and scholars ever since first viruses were identified at the turn of the 19th century and, with the discovery of complex 'giant viruses' (7) and 'huge phages' (8), it is as pertinent as ever (4-9). The traditional criteria for differentiating viral lineages from cellular lineages based on size, or on genetic, proteomic and metabolic complexity, are no longer valid and impede progress; indeed, absent these obsolete criteria, we might have learned about the viral nature of "small cocci bacteria," "endocytobionts," and "archaeal endosymbionts" (eventually identified as mimiviruses, pandoraviruses, and pithoviruses, respectively), many years, if not decades earlier. 
In a 'Behind the Paper' narrative (10), Ricardo Cavicchioli mentioned that Antartic Nanohaloarchaeota were discovered accidentally while searching for viruses infecting haloarchaea. Unknowingly, though, Hamm et al. discovered a true 'viral treasure trove,' as Antartic Nanohaloarchaeota represent promising experimental models for the fusion theory on the origin of incipient/ancestral viral lineages $(4,6)$.
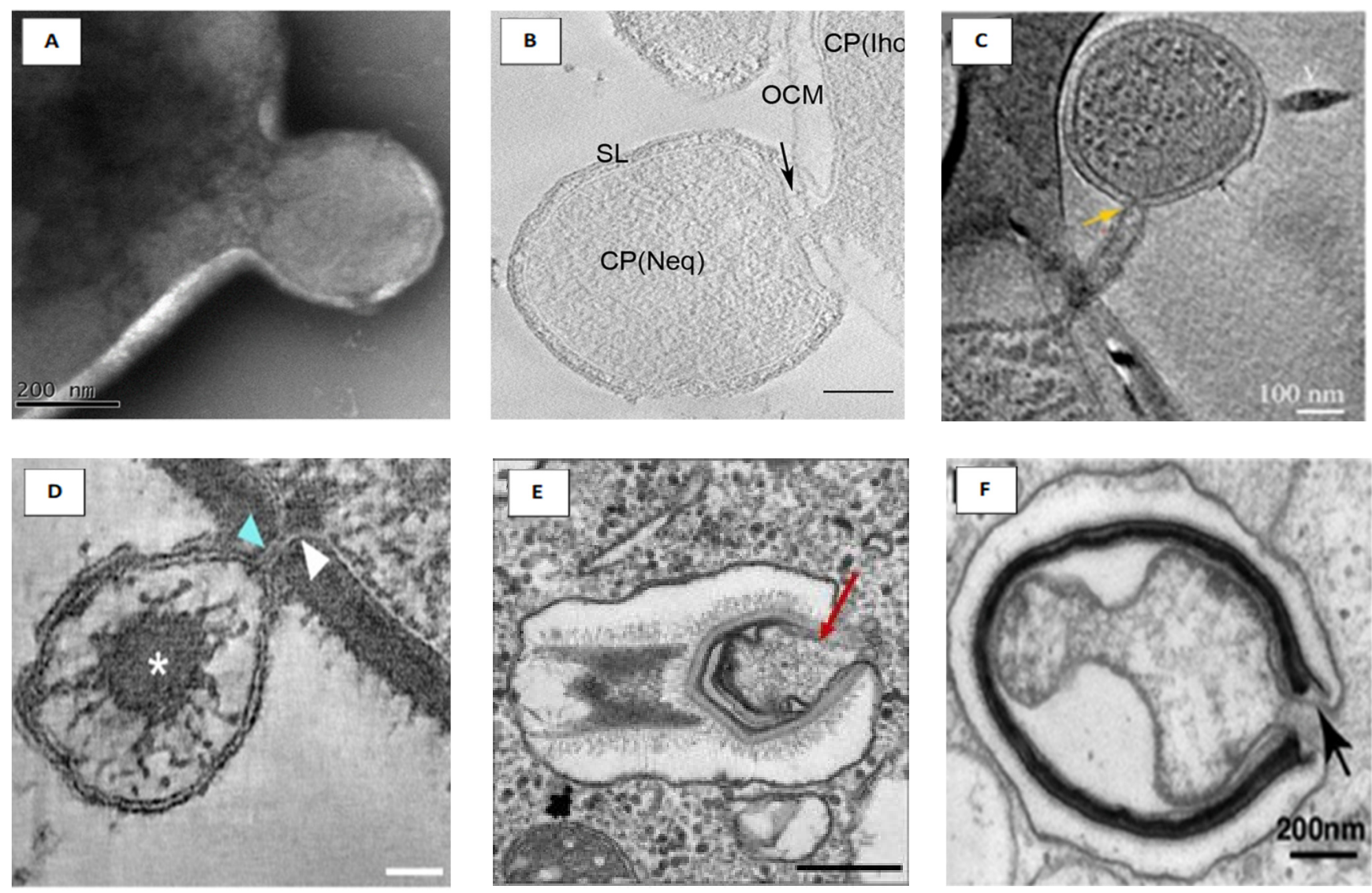

Fig. 1. Parasite-Host Cell Fusion. Top panel: archaea - archaea fusion. Lower panel: eukaryal viruses - eukaryal cells fusion.

(A) Ca. Nha. antarcticus fusion with Hrr. lacusprofundi. TEM image showing the putative fusion of $C a$. Nha. antarcticus (small cell) and Hrr. lacusprofundi (large cell); this and other images in Hamm et.al. suggest: "cell association possibly involving extracellular material (Fig. 6 E and F and SI Appendix, Figs. S1 A-D and S2 E and F), cell fusion suggestive of a shared lipid membrane (Fig. 6 B-D and SI Appendix, Fig. S7 G and H), and possible membranecollar structures associated with budding/fission"; "Small cell that appears to be attached with a possible membrane collar to a large cell (E and F); the morphology is reminiscent of a site of budding (detachment) or possibly, initial stages of fusing after attachment" (image and text excerpts from Ref. 1).

(B) N. equitans fusion with I. hospitalis. Tomogram showing the fusion of N. equitans and I. hospitalis membranes, allowing direct connection between the cytoplasm of both organisms (OCM, outer cellular membrane; SL, S-Layer; CP, cytoplasm; Iho, I. hospitalis; Neq, N. equitans); scale bar $100 \mathrm{~nm}$ (image from Ref. 3).

(C) ARMAN fusion with Thermoplasmatales. 3D cryo-ET reconstruction imagine showing a cytoplasmic bridge (yellow arrow), conducive to transfer of metabolites and other components, between a Thermoplasmatal cell and an ARMAN cell; an elongated virus particle $(\mathrm{V})$ representing a typical viral lineage infecting archaea is also visible (hypothetically, their ancestors were more complex viral lineages that originated from DPANN-like parasitic lineages and diversified into a myriad of viruses by reductive evolution) (image from Ref. 11).

(D) Paramecium bursaria chlorella virus-1 fusion with a chlorella cell. STEM tomogram showing the fusion of Paramecium bursaria chlorella virus-1 membrane with the membrane of chlorella host cell, which generates a tunnel for the 
transfer of the viroplasm containing the genome; the membrane tunnel and the protrusion of the host membrane are marked with blue and white arrowheads, respectively; asterisk represents the viral genome; scale bar: 50 nm); (image from Ref. 12).

(E) Tupanvirus fusion with Acanthamoeba castellanii. TEM image showing the inner membrane of Tupanvirus fused with the host's phagosome membrane (red arrow) leading to transfer of viroplasm containing the viral genome into the host cell, which is an essential environment for most stages in the viral life cycle; scale bar $450 \mathrm{~nm}$ (image from Ref. 13).

(F) Pandoravirus fusion with Acanthamoeba. Electron microscope image of Pandoravirus membrane fused with the vacuole membrane (arrow) and transfer of viroplasm containing the genome into the host cytoplasm (image from Ref. 14).

\section{References}

1. J.N. Hamm et al., Unexpected host dependency of Antarctic Nanohaloarchaeota. Proc Natl Acad Sci U S A. doi: 10.1073/pnas.1905179116. (2019).

2. N. Dombrowski, J.H. Lee, T.A. Williams, P. Offre, Spang A., Genomic diversity, lifestyles and evolutionary origins of DPANN archaea. FEMS Microbiol Lett. 366, (2019).

3. T. Heimerl et al., A complex endomembrane system in the archaeon Ignicoccus hospitalis tapped by Nanoarchaeum equitans. Front Microbiol.8:1072 (2017).

4. C.I. Bandea, A new theory on the origin and the nature of viruses. J. Theor. Biol. 105, 591-602 (1983).

5. C.I. Bandea, The Origin and Evolution of Viruses as Molecular Organisms. Nature Precedings: http://precedings.nature.com/documents/3886/version/1 (2009).

6. C.I. Bandea, Evolution of giant viruses from larger ancestors. Comment on "Virus genomes from deep sea sediments expand the ocean megavirome and support independent origins of viral gigantism" by D. Bäckström et al., bioRxiv 469403; doi: https://doi.org/10.1101/469403 (2018).

7. P. Forterre, Giant viruses: conflicts in revisiting the virus concept. Intervirology. 53(5):362-378 (2010).
8. B. Al-Shayeb et al., Clades of huge phage from across Earth's ecosystems.

bioRxiv 572362; doi: https://doi.org/10.1101/572362 (2019).

9. V. Racaniello, The virus and the virion. Virology Blog; http://www.virology.ws/2010/07/22/the-virus-andthe-virion/ (2010).

10. R. Cavicchioli, Behind the Paper: Discovery, hypothesis \& Koch's postulates - Nanohaloarchaeota are NOT free-living. Nature Research Communities Microbiology; https://go.nature.com/2IQ3Fge (2019).

11. L.R. Comolli, J.F. Banfield, Inter-species interconnections in acid mine drainage microbial communities. Front Microbiol. 5:367 (2014).

12. E. Milrot et al., Virus-host interactions: insights from the replication cycle of the large Paramecium bursaria chlorella virus. Cell Microbiol. 18:3-16 (2016).

13. J. Abrahão et al., Tailed giant Tupanvirus possesses the most complete translational apparatus of the known virosphere. Nat Commun. 9:749. (2018).

14. N. Philippe et al., Pandoraviruses: amoeba viruses with genomes up to $2.5 \mathrm{Mb}$ reaching that of parasitic eukaryotes. Science. 341:281-286. (2013). 